$\mathbb{T}$ periodica polytechnica

Mechanical Engineering

$56 / 1(2012) 49,53$

web: http://www.pp.bme.hu/me

(c) Periodica Polytechnica 2012

RESEARCH ARTICLE

\section{Vibrator with DC motor driven eccentric} rotors

\author{
Ákos Miklós / Zsolt Szabó
}

Received 2012-04-30

\begin{abstract}
DC motor drives are widely used in many devices in mechatronics and industry. Since the performance of these motors depends on their angular velocity, there are some interesting phenomena originating from the counter-action of the drive and the driven mechanical system.

The present work proposes a four-degree-of-freedom mechanical model with two DC motor driven eccentric rotors. Only translational in-plane motion of the base is considered.

Based on the stationary motions of the proposed system, the changed DC motor performance and the phenomenon of selfsynchronization have been showed analytically and by some simulation results.
\end{abstract}

\section{Keywords}

self-synchronization $\cdot$ DC motor $\cdot$ dual excenter $\cdot$ vibromotor

\section{Ákos Miklós}

Department of Applied Mechanics, BME, H-1111, Budapest, 5 Múegyetem rkp., Hungary

e-mail: miklosa@mm.bme.hu

\section{Zsolt Szabó}

Department of Applied Mechanics, BME, H-1111, Budapest, 5 Múegyetem rkp., Hungary

e-mail: szazs@mm.bme.hu

\section{Introduction}

Mechanical systems with electric motor driven eccentric rotors are widely used in mechatronics and in the industry. Common applications are industrial shakers and vibromotors of compacting machines, conveyors etc. Of course, in some devices unwanted eccentricity occurs as well, such as in optical drives or centrifuges.

Self-synchronization of such systems occurs, if the rotating speed of more rotors becomes equal. This synchronization can be generated by the driving voltage, by control methods of driving motors, or by auto-synchronization of the eccentric rotors [1]. Auto-synchronization or self-synchronization is caused by mechanical coupling between subsystems. Its first scientific mention was made by Christiaan Huygens in the $17^{\text {th }}$ century [2], who experienced the synchronization of pendulum clocks.

Since self-synchronization is required in some systems (e.g. industrial shakers with two rotors, that are turning in opposite direction), and has to avoided in some other cases (e.g. industrial machines grounded on the same basement, where selfsynchronization can lead to heavy overloads of the industrial building), investigation of this mechanical synchronization is essential.

Since the work of Huygens the synchronization phenomena attracted the attention of many scientists. In the work of Blekhman et al. [3] a general definition of the selfsynchronization is given, and some applications and numerical simulations are showed. Nijmeijer reviewed the synchronization from a dynamical control perspective [4]. Wen et al. developed a mechanical model with two eccentric rotors rotating in the same direction, and showed a control method to achieve synchronization [5]. They also investigated stability and bifurcation behaviour of self-synchronization [6].

The aim of this paper is to describe a mechanical model with two eccentric and coaxial rotors, which are driven by DC motors. The model considers only plane motion of the base mass, which the rotors are attached to. The paper shows two phenomena, the self-synchronization in this system, and the changed performance of the DC motors near to the resonance of the mechanical system. 
After the Introduction, Section 2 describes the developed mechanical model, the equations of motion and the stationary solution of the equations of motion. Then, in Section 3 and 4 the behaviour near to the resonance and the self-synchronization of the system are showed, respectively. In Section 5 some conclusions are summarized for the investigated model.

\section{Mechanical model and equations of motion othe twin-rotor system}

In Fig. 1 the moving mass $m$ is modelled, which is excited by two eccentric rotors. The mass is suspended isotropic with spring stiffness $k$ and damping coefficient $c$ in both planar directions. The position of the centre of the moving mass $C$ is described by the polar coordinates $a$ and $\vartheta$ with respect to the fixed point $O$ of the absolute system. The rotors are driven by DC motors with same electrical and mechanical parameters. The motors are acting with torques $T_{1}$ and $T_{2}$ on the rotors. The mass, the mass moment of inertia and the eccentricity of the rotors are $m_{0}, J_{0}$ and $e$, respectively. The angular positions of the rotors can be described by the angular position of the common bisector $\varphi$, and the half of the phase angle between the rotors $\delta$.

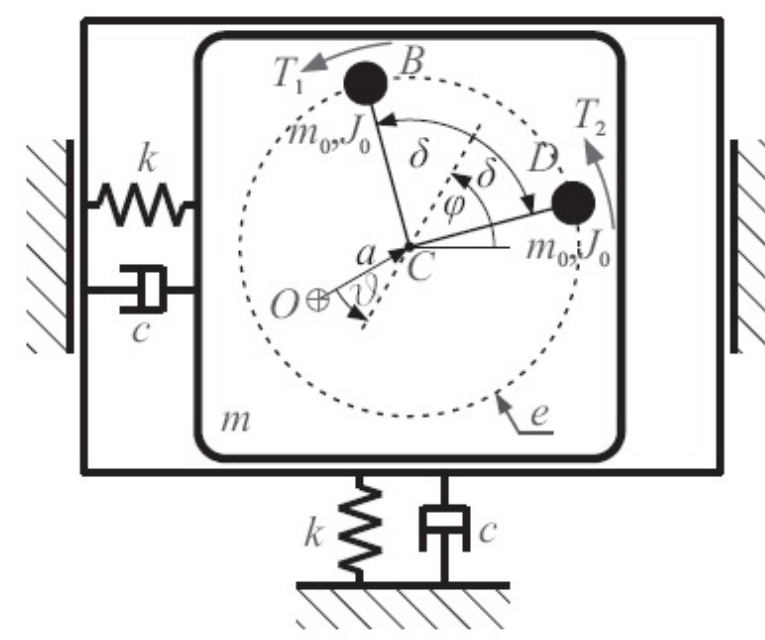

Fig. 1. Mechanical model with two eccentric rotors

So we get a four-degree-of-freedom model, where we calculate the torques of the DC motors from the stationary motor performance:

$$
T_{i}=k_{t} \frac{u_{i}(t)-k_{e} \omega_{i}(t)}{R} .
$$

In Equation (1) $k_{t}, k_{e}$ and $R$ are the torque constant, the speed constant and the electric resistance of the motor, respectively, $u$ is the driving voltage and $\omega$ is the angular velocity of the rotor. Considering parameter sets of common mechatronical systems, the electrical dynamics of the DC motors can be neglected, since electric transients decay much faster than mechanical ones.

\subsection{Equations of motion}

We obtain the equations of motion by the Lagrangian equation of the second kind. The kinetic- and potential energy of the system and the power of the external forces are given by the following forms:

$$
\begin{gathered}
E_{k i n}=\frac{1}{2}\left(m v_{C}^{2}+m_{0}\left(v_{B}^{2}+v_{D}^{2}\right)+J_{0}\left(\omega_{B}^{2}+\omega_{D}^{2}\right)\right), \\
E_{p o t}=\frac{1}{2} s a^{2} \\
P=T_{1}(\dot{\varphi}+\dot{\delta})+T_{2}(\dot{\varphi}-\dot{\delta})-c v_{C}^{2} .
\end{gathered}
$$

Considering, that

$$
\begin{aligned}
& r_{O C}=a[\cos (\varphi-\vartheta), \sin (\varphi-\vartheta)], \\
& r_{C B}=e[\cos (\varphi+\delta), \sin (\varphi+\delta)], \\
& r_{C D}=e[\cos (\varphi-\delta), \sin (\varphi-\delta)],
\end{aligned}
$$

and

$$
\begin{aligned}
v_{B}^{2} & =\left(\dot{r}_{O C}+\dot{r}_{C B}\right) \times\left(\dot{r}_{O C}+\dot{r}_{C B}\right) \\
v_{D}^{2} & =\left(\dot{r}_{O C}+\dot{r}_{C D}\right) \times\left(\dot{r}_{O C}+\dot{r}_{C D}\right) v_{C}^{2}=\dot{r}_{O C} \times \dot{r}_{O C} ; \\
\omega_{B} & =\dot{\varphi}+\dot{\delta} ; \omega_{D}=\dot{\varphi}-\dot{\delta} .
\end{aligned}
$$

After that, we can derive the dimensionless equations of motion:

$A^{\prime \prime}-\Phi^{\prime \prime} \cos \Delta \sin \Theta-\Delta^{\prime \prime} \sin \Delta \cos \Theta=A\left(\Phi^{\prime}-\Theta^{\prime}\right)^{2}$

$-2 \zeta A^{\prime}-A+\left(\Phi^{\prime 2}+\Delta^{\prime 2}\right) \cos \Delta \cos \Theta$

$-2 \Phi^{\prime} \Delta^{\prime} \sin \Delta \sin \Theta$,

$\Theta ” A-\Phi ”(A+\cos \Delta \cos \Theta)+\Delta " \sin \Delta \sin \Theta=$

$-2 \Phi^{\prime} \Delta^{\prime} \sin \Delta \cos \Theta 2\left(\Phi^{\prime}-\Theta^{\prime}\right)\left(\zeta A+A^{\prime}\right)-$

$\left(\Phi^{\prime 2}+\Delta^{\prime 2}\right) \cos \Delta \sin \Theta$

$-A " \cos \Delta \sin \Theta-\Theta ”\left(A^{2}+A \cos \Delta \cos \Theta\right)$

$-A \Delta " \sin \Delta \sin \Theta+\Phi ”\left(1+A+2 A \cos \Delta \cos \Theta+k_{1}\right)=$

$2 A \Phi^{\prime} \Delta^{\prime} \sin \Delta \cos \Theta+$

$+2\left(\Theta^{\prime}-\Phi^{\prime}\right)\left(\zeta A^{2}+A A^{\prime}+A^{\prime} \cos \Delta \cos \Theta\right)-$
$-A\left(\Theta^{\prime 2}-2 \Theta^{\prime} \Phi^{\prime}-\Delta^{\prime 2}\right) \cos \Delta \sin \Theta+\Sigma U-\kappa \Phi^{\prime}$,

$A \Theta " \sin \Delta \sin \Theta-A " \sin \Delta \cos \Theta-A \Phi ” \sin \Delta \sin \Theta+$

$\Delta " k_{1}=\Delta U$

$2 A^{\prime}\left(\Phi^{\prime}-\Theta^{\prime}\right) \sin \Delta \sin \Theta-A\left(\Theta^{\prime}-\Phi^{\prime}\right)^{2} \sin \Delta \cos \Theta-\kappa \Delta^{\prime}$,

where primes denote derivatives with respect to the dimensionless time $\tau$. The dimensionless generalized coordinates and input voltages are as follows:

$$
\begin{gathered}
a(t)=L_{0} A(\tau), \vartheta(t)=\Theta(\tau), \\
\varphi(t)=\Phi(\tau), \delta(t)=\Delta(\tau), \\
\Sigma U(\tau)=c_{u}\left[u_{1}(t)+u_{2}(t)\right], \\
\Delta U(\tau)=c_{u}\left[u_{1}(t)-u_{2}(t)\right] .
\end{gathered}
$$


The parameters used for the dimensionless equations are:

$$
\begin{aligned}
\tau & =\alpha t, \\
\zeta & =\frac{c}{2 \alpha\left(m+2 m_{0}\right)}, \\
\alpha & =\sqrt{\frac{k}{m+2 m_{0}}}, \\
L_{0} & =\frac{2 e m_{0}}{m+2 m_{0}}, \\
k_{1} & =\frac{m+2 m_{0}}{2 e^{2} m_{0}^{2}}\left(e^{2} m_{0}+J_{0}\right), \\
c_{u} & =\frac{k_{t}\left(m+2 m_{0}\right)}{4 e^{2} m_{0}^{2} \alpha^{2} R}, \\
\kappa & =\frac{k_{t} k_{e}\left(m+2 m_{0}\right)}{2 e^{2} m_{0}^{2} \alpha R} .
\end{aligned}
$$

Here we can see, that $\alpha$ and $\zeta$ are natural angular frequency and damping ratio of the suspended mass $m . L_{0}$ is the distance of the common centre of mass from point $C$ if the phase angle between the two rotors is zero.

From the equations of motion one can see, that the dynamic behaviour depends on three parameters: on the damping ratio $\zeta$, on $k_{1}$, which comes from the mass moment of inertia of the rotors and on $\kappa$, which contains electrical parameters of the driving motors.

\subsection{Stationary motions}

As self-synchronization can only occur, if the system achieves its stationary state, we have to investigate stationary motions of the described model. Assuming, that the stationary values of the generalized coordinates $A_{0}, \Theta_{0}$ and $\Delta_{0}$ are constant, respectively, and the first derivative of $\Phi$ is constant $\lambda$, we get the following equations:

$$
\begin{gathered}
\lambda^{2} A_{0}-A_{0}+\lambda^{2} \cos \Delta_{0} \cos \Theta_{0}=0, \\
2 \zeta A_{0} \lambda-\lambda^{2} \cos \Delta_{0} \sin \Theta_{0}=0, \\
-2 \zeta A_{0}^{2} \lambda+\Sigma U-\kappa \lambda=0 \\
-A_{0} \lambda^{2} \sin \Delta_{0} \cos \Theta_{0}+\Delta U=0 .
\end{gathered}
$$

This means, that the phase angle between the two rotors and the common angular velocity are constant, and the motion of the base follows the motion of the rotors with some phase delay and constant amplitude.

We consider, that in the dimensionless case the first derivative of $\Phi$ equals to the frequency ratio of the eccentric excited system, so we can use instead of $\Phi^{\prime}$ the frequency ratio $\lambda=\varphi^{\prime} / \alpha$. From equations (eq8.a-b) we can derive the amplitude and phase delay of the motion of point $C$ of the moving mass:

$$
\tan \Theta_{0}=\frac{2 \zeta \lambda}{1-\lambda^{2}}
$$

$$
A_{0}=\frac{\lambda^{2} \cos \Delta_{0}}{\sqrt{\left(1-\lambda^{2}\right)^{2}+(2 \zeta \lambda)^{2}}},
$$

which are the well known formulae for eccentric excited oscillations [7]. Furthermore, we can derive the formulae for the sum and difference of the input voltages, which we need to achieve the stationary motion for a given frequency ratio and phase shift:

$$
\begin{aligned}
& \Sigma U=\frac{2 \zeta \lambda^{5} \cos ^{2} \Delta_{0}}{\left(1-\lambda^{2}\right)^{2}+(2 \zeta \lambda)^{2}}+c \lambda, \\
& \Delta U=\frac{\lambda^{4}\left(1-\lambda^{2}\right) \cos \Delta_{0} \sin \Delta_{0}}{\left(1-\lambda^{2}\right)^{2}+(2 \zeta \lambda)^{2}}
\end{aligned}
$$

From equations 10 and 11 it is obvious, that for every frequency ratio and phase shift there exists a stationary motion. Fig. 2 and Fig. 3 shows how the dynamic behaviour depends on the parameters $\Delta_{0}$ and $\lambda$. The characteristic peek at $\lambda=1$ in Fig. 2 decreases with growing damping of the suspension.

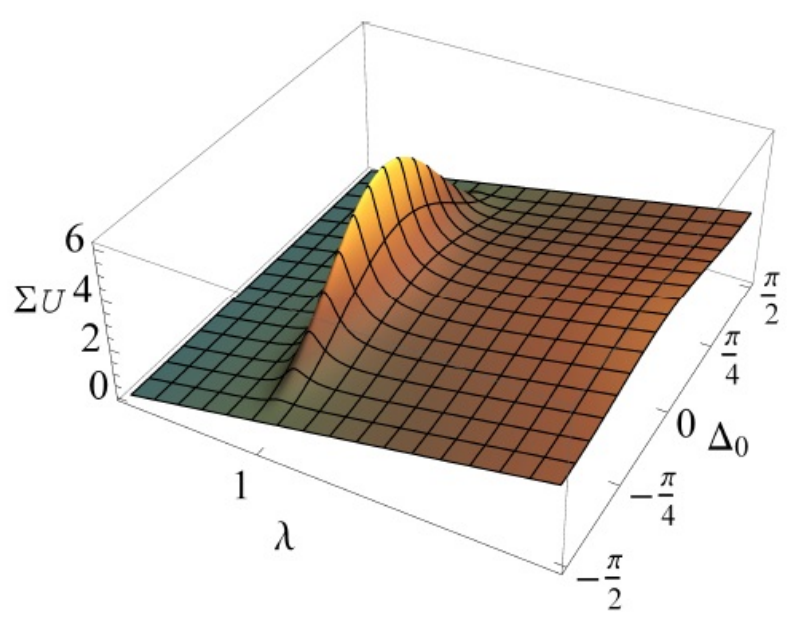

Fig. 2. Sum of the driving voltages in stationary case $(\zeta=0.1$ and $\kappa=1$

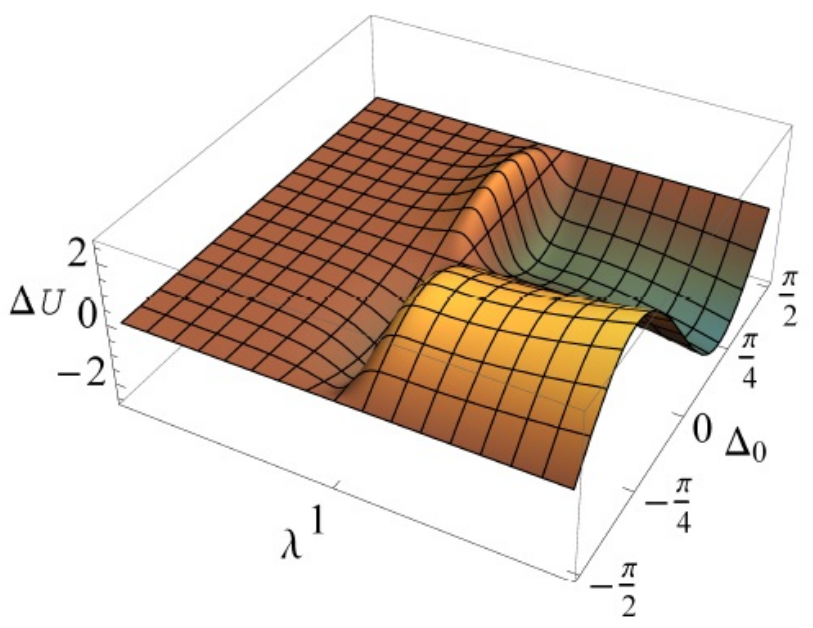

Fig. 3. Driving voltage difference in stationary case $(\zeta=0.1$ and $\kappa=1)$

\section{DC motor performance in resonance}

Eq. 10 explains an important mechanical phenomenon. If a DC motor with eccentric rotor is driven over resonance, the 
high power consumption of the vibrations near to the resonance hinders the speed up of the rotor. Furthermore, if the driving voltage of the DC motor is limited, higher angular velocities possibly cannot be reached. In the presented mechanical model the $\Delta_{0}=0$ case corresponds to the one rotor system. In Figure 4 a simulation is showed for this case, where the phase shift is fixed to zero. The driving voltages are increased to drive the rotors over the resonance frequency, and then decreased to zero.
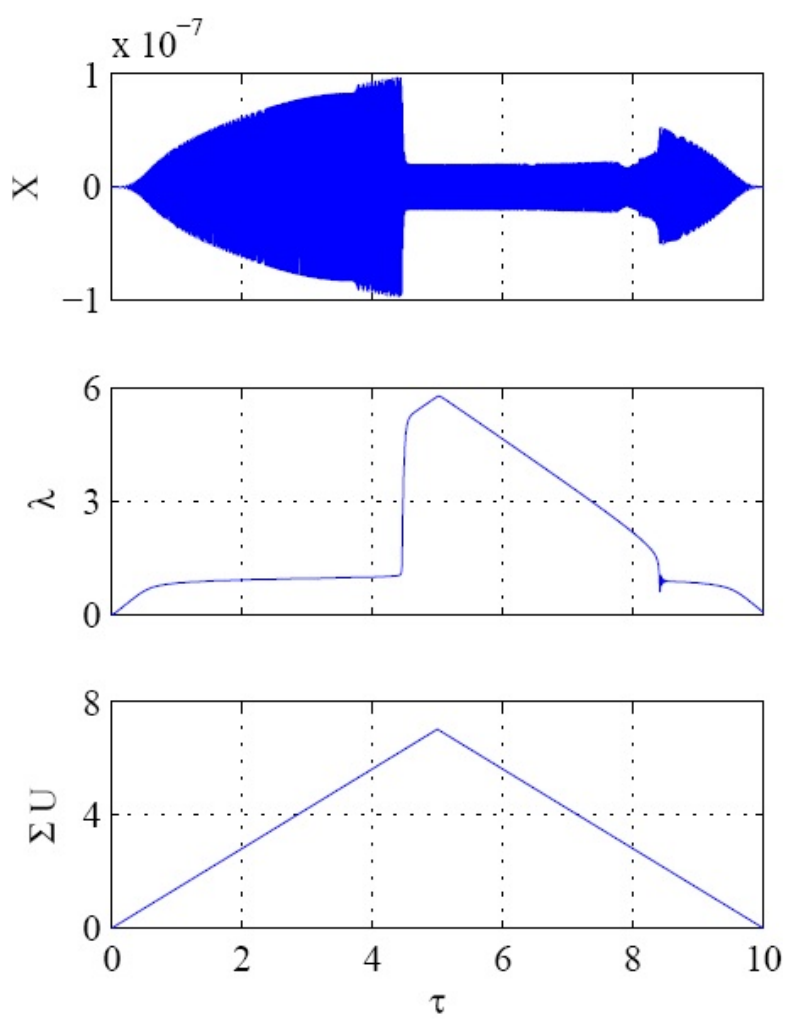

Fig. 4. Time history of vibrations of eccentric DC motor over resonance $\left(\zeta=0.1, \kappa=1\right.$ and $\left.k_{1}=1903\right)$

In Fig. 4 the first panel shows the vertical dimensionless displacement of the moving mass. The amplitude of the vibration shows a nonlinear-oscillator-like behaviour, however the system does not contain any nonlinear element. This could be important for systems, where nonlinear behaviour with unknown reason is experienced.

Fig. 5 shows the same simulation results comparing to the analytical solution of the stationary motions. Here we can see, that in a given range for one voltage sum value there exists one stable and one unstable stationary motion. After reaching the positive or negative peek voltage sum the system is jumping from one to the other stable solution.

\section{Self-synchronization}

Without investigating the stability of the stationary motions given by equations (10) and (11) there are some general facts.

If the difference between driving voltages is zero, below the resonance frequency the system tends to stabilize itself at $\Delta_{0}=0$, where the rotors are in the same phase. Above the res-

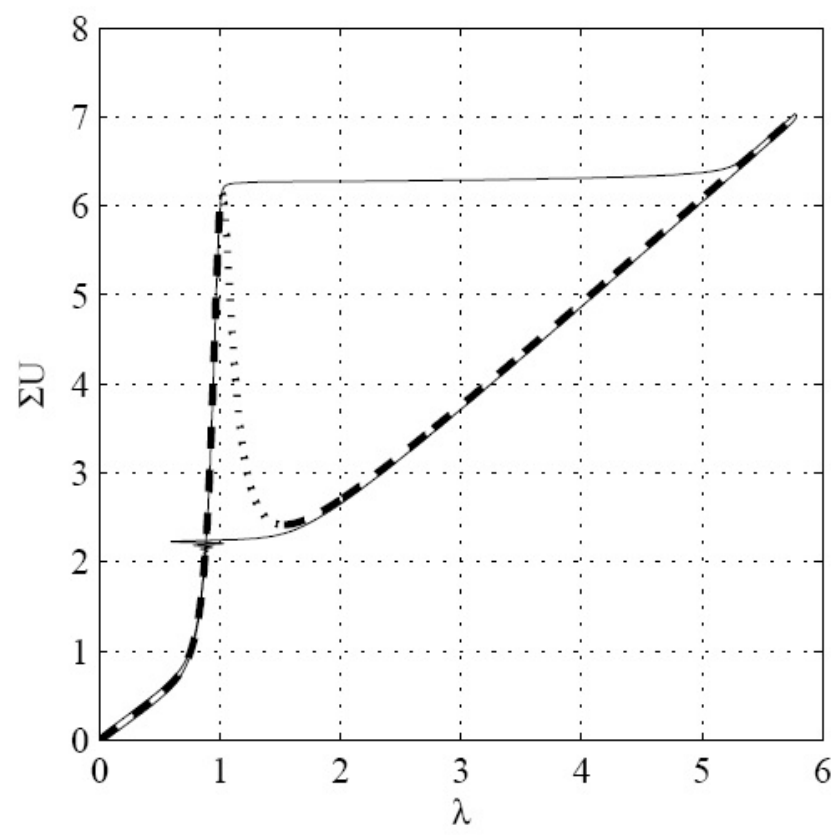

Fig. 5. Changed motor performance over resonance (dashed line - stable stationary motion; dotted line - unstable stationary motion; thin line - simulation result)
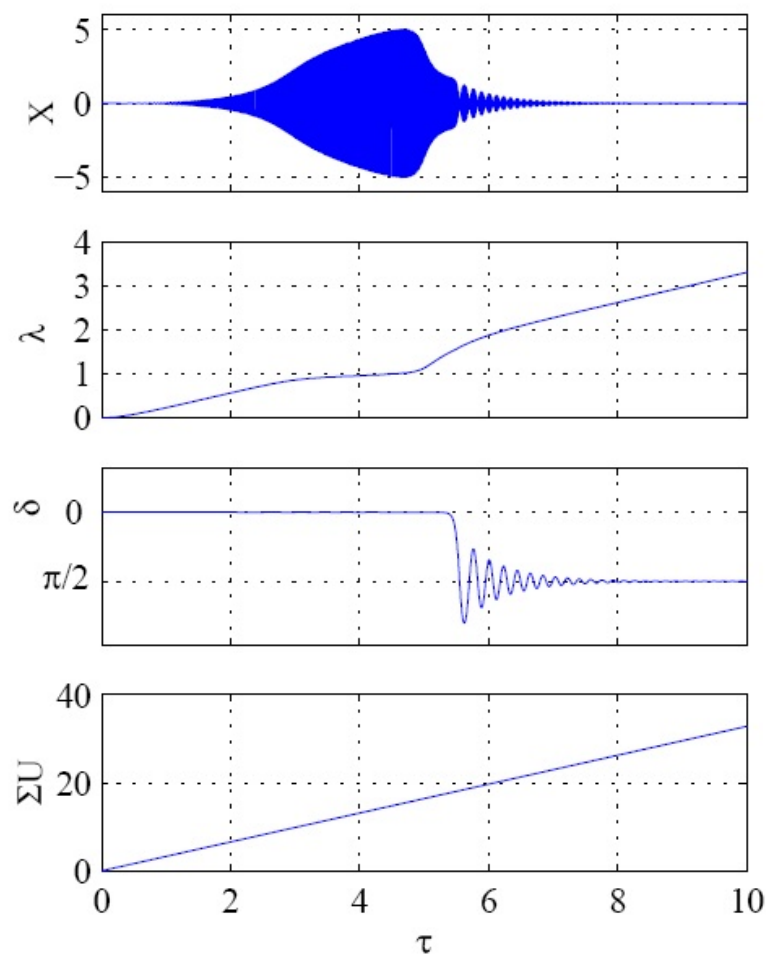

Fig. 6. Displacement, frequency ratio and phase angle for equal driving voltages below and above resonance $\left(\zeta=0.1, \kappa=9.6, k_{1}=1903\right.$ and $\left.\Delta U=0\right)$

onance frequency, the motion is stable at $\Delta_{0}=\pi / 2$, where the rotors are in opposite phase. In Fig. 6, simulation results can be seen for equally increasing driving voltages. After reaching and exceeding resonance frequency, the phase shift $\delta$ is jumping from zero to $-\pi / 2$ with some transient. These observations 

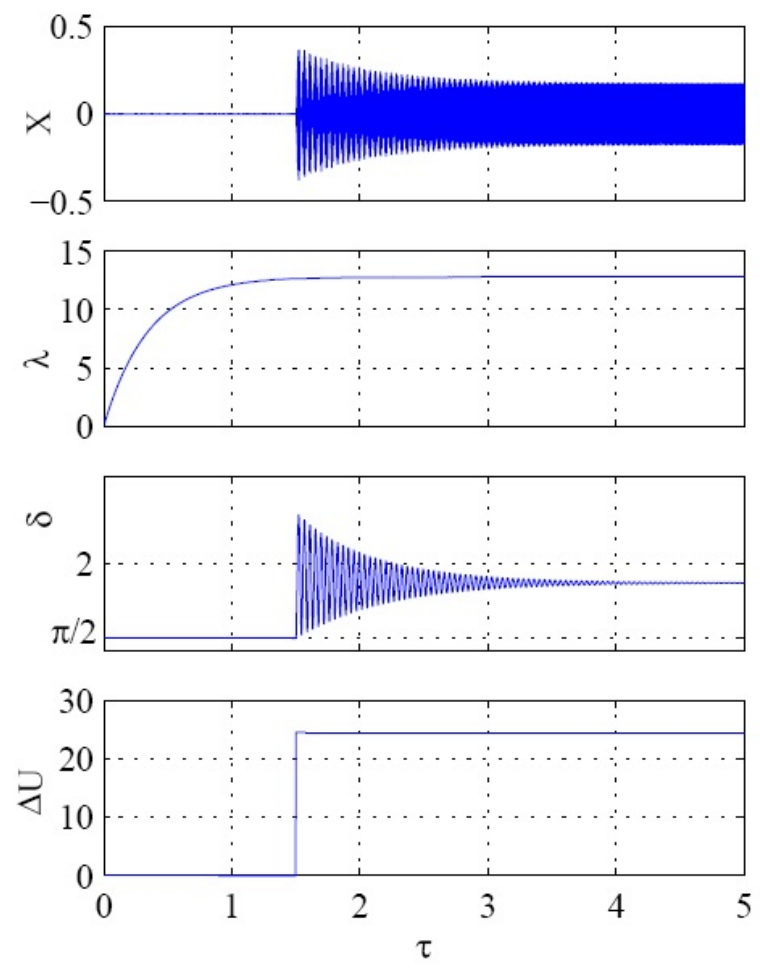

Fig. 7. Displacement, frequency ratio and phase angle for different driving voltages above resonance $\left(\zeta=0.1, k=9.6, k_{1}=1903\right.$ and $\left.\Sigma U=123\right)$

correspond to the behaviour of passive ball balancers [1].

In case of nonzero difference between driving voltages the stationary phase shift between the rotors can be adjusted, however at one point the system becomes unstable, and the self synchronization disappears. In Fig. 7 the effect of nonzero driving voltage difference is showed over resonance frequency. The robustness of the stability of the stationary motions depends on the system parameters $\zeta, k_{1}$, and $\kappa$, and on the working point, which is characterized by $\lambda$ and $\Delta_{0}$. In Fig.7 the time history of a simulation with nonzero difference between driving voltages is showed. At the point, where the voltage difference becomes nonzero, the phase difference is jumping after some transient from $\pi / 2$ to another constant value.

\section{Conclusions}

In this paper, a mechanical model was showed with two, DC motor driven eccentric rotors, exciting an in-plane moving mass. Considering the stationary motions of the system we showed, that the DC motor performance changes over resonance, and in some cases the power consumption of vibrations can hinder the speed up of eccentric rotors. Furthermore, we proved that there exists a stationary motion for every phase angle and synchronous angular velocity of the rotors. The stability of these stationary motions depends on the system parameters, phase angle and frequency ratio. Generally, the system tends to stabilize itself below the resonance frequency of the base mass at zero phase angle, and above the resonance at $\Delta_{0}=\pi / 2$. Thus, it shows some analogy with the passive automatic ball balancer.

\section{References}

1 Leonov GA, Phase Synchronization: Theory and Applications, Automation and Remote Control 67 (2006), no. 10, 1573-1609, DOI 10.1134/S0005117906100031.

2 Huygens C, Horoloqium Oscilatorium, Paris, 1673.

3 Blekhman I., On self-synchronization and controlled synchronization, Systems \& Control Letters 31 (1997), 299-305, DOI 10.1109/COC.1997.633464.

4 Nijmeijer H, A dynamical control view on synchronization, Physica D: Nonlinear Phenomena 154 (2001), no. 3-4, 219-228, DOI 10.1016/S01672789(01)00251-2.

5 Liu X, Observation and Control of Phase Difference for a Vibratory Machine of Plane Motion, 2010 International Conference on Computer Mechatronics, Control and Electronic Engineering (CMCE) 4, DOI 10.1109/CMCE.2010.5610130.

6 Han QK, Stability and Bifurcation of Self-synchronization of a Vibratory Screener Excited by Two Eccentric Motors, Adv. Theor. Appl. Mech 1 (2008), no. 3, 107-119.

7 Ludvig Gy, Gépek dinamikája, Múszaki Könyvkiadó, 1986. 